\title{
ESTABILIDADE MICROBIOLÓGICA, FÍSICO-gUÍMICA E SENSORIAL DE PEDÚNCULOS DE CAJU (Anacardium occidentale L.) PROCESSADOS POR MÉTODOS COMBINADOS ${ }^{1}$
}

\author{
Patricia Campos MESQUITA ${ }^{2}$, Geraldo Arraes MAIA ${ }^{3}$,Men de Sá Moreira de SOUZA FILHO ${ }^{4}$,Renata Tieko NASSU ${ }^{4}$
}

\section{RESUMO}

Os pedúnculos de caju processados por métodos combinados e armazenados à temperatura ambiente $\left(28^{\circ} \mathrm{C}\right)$ foram avaliados quanto à tendência a mudanças físico-químicas, microbiológicas e sensoriais. Os resultados confirmam que os tipos de obstáculos usados (redução da Aw, tratamento térmico brando, redução do pH, adição de ácido ascórbico, benzoato de sódio a 1000 ppm e $\mathrm{SO}_{2}$ a 600 e 900 ppm) e sua intensidade foram capazes de assegurar a estabilidade microbiológica do produto durante a armazenagem à temperatura ambiente por 120 dias, bem como uma boa aceitação sensorial.

Palavras-chave: estabilidade; pendúnculo de caju; métodos combinados.

\section{SUMMARY}

MICROBIOLOGICAL, PHYSICO-CHEMICAL AND SENSORIAL STABILITY OF CASHEW APPLES (Anacardium occidentale L.) PROCESSED BY COMBINED METHODS. Cashew apples processed by combined methods were stored at room temperature $\left(28^{\circ} \mathrm{C}\right)$ in order to evaluate the tendency for chemical, microbiological and sensorial changes during 120 days of storage. Results confirmed that the obstacles used (reduction of water activity, mild heat treatment, pH reduction, ascorbic acid addition, 1000ppm sodium benzoate, 600 and $900 \mathrm{ppm}$ of $\mathrm{SO}_{2}$ ) and their intensities were capable to assure the microbiological stability and sensorial acceptance of the product during storage stability at room temperature for 120 days.

Keywords: stability; cashew apple; combined methods.

\section{1 - INTRODUÇÃO}

A produção nacional de pedúnculos de caju no ano de 1998 foi estimada em 1.450.000 toneladas métricas [7]. O seu aproveitamento é de menos de $6 \%$ da produção nacional de pedúnculo [18]. A alta perecibilidade, juntamente com a falta de facilidade na armazenagem durante os meses de pico do processamento industrial, contribui para esta perda física. Conseqüentemente há grande expectativa para o desenvolvimento de processos para conservação local (junto ao produtor) de frutas por métodos combinados [2].

A tecnologia de processamento minimo/métodos combinados é um método moderno que estende a vida-deprateleira de alimentos, permitindo sua melhor distribuição. Esta tecnologia encontra demanda de consumo pela conveniência e pela qualidade do gosto fresco. Pode ser aplicada a vários estágios da cadeia de distribuição do alimento, na estocagem, no processamento e/ou embalagem [17]. Segundo LOPEZ-MALO et al. [14], frutos processados por este método podem ser consumidos ou usados como insumos em confeitaria, produtos de padaria, produtos de laticínio, compotas, geléias e gelatinas.

Produtos de frutas preservadas pelo método de barreiras ou método combinado têm um potencial de mercado muito grande devido a sua melhor retenção do sabor e

\footnotetext{
1. Recebido para publicação em 11/10/2001. Aceito para publicação em 31/03/2003 (000761).

${ }^{2}$ Universidade Federal do Ceará. E-mail: Paty mesquita@hotmail.com ${ }^{3}$ Departamento de Tecnologia de Alimentos - Universidade Federal do Ceará. Av. Mister Hull 2977 Bloco 857 Alagadiço, CEP 60356000 Fortaleza-CE.

${ }^{4}$ EMBRAPA - Agroindústria Tropical - Rua Sara Mesquita 2270 - Bairro Pici - CEP: 60511110 - Fortaleza-CE

* A quem a correspondência deve ser enviada.
}

textura originais quando comparados a produtos desidratados [10], aos novos requerimentos de produtos minimamente processados e estabilidade à temperatura ambiente [6, 13].

A base da tecnologia de métodos combinados (MC) é a exposição simultânea das células microbianas vegetativas a vários fatores adversos (redução da Aw, decréscimo do $\mathrm{pH}$, adição simples ou combinada de agentes antimicrobianos, tratamento térmico brando etc), de forma que o conteúdo de energia consumida nos processos homeostáticos é elevado e as células microbianas não têm suficiente energia disponivel para o desenvolvimento e reprodução, e assim morrem [11, 12].

A aplicação desta tecnologia em frutas pode ser limitada pelo fator sensorial, devido ao uso de altas concentrações de soluto ou ao alto grau de secagem requerido para redução da Aw que assegurará a estabilidade microbiológica [3].

No contexto da tecnologia de barreiras, uma interessante alternativa aos PFUI (Produtos de Fruta de Umidade Intermediária), são os Produtos de Fruta de Alta Umidade (PFAU) $(\mathrm{Aw}>0,92)$ desenvolvida por SAJUR [21], ALZAMORA et al.[1] e ARGAIZ et al.[5]. Nestes produtos, o controle da atividade de água (Aw) não é mais a principal barreira que proporciona estabilidade, mas sim a combinação dos fatores, tais como tratamento térmico, leve redução do $\mathrm{pH}$ e adição de antimicrobianos permitidos, resultando em produtos com vida-de-prateleira estável apresentando atrativas características parecidas com produtos frescos [3].

Este trabalho teve como objetivo, gerar conhecimentos básicos sobre a conservação do pedúnculo do caju por métodos combinados, utilizando a tecnologia de obstáculos, como alternativa aos processos de conservação 
tradicionais e onerosos (congelamento, refrigeração, atmosfera controlada), buscando alcançar uma redução de perdas pós-colheita de frutos, aumentando o valor agregado da matéria-prima e assegurando adequada utilização da capacidade instalada nas indústrias de conservação.

\section{2 - MATERIAL E MÉTODOS}

Foi utilizado como matéria-prima de pesquisa o pedúnculo de caju (Anacardium occidentale, L.) obtido nos campos experimentais de Pacajus e Paraipaba, Ceará, do CNPAT/EMBRAPA.

Os pedúnculos foram submetidos ao processamento (conforme fluxograma - Figura 1) em xarope de sacarose a $25^{\circ} \mathrm{Brix}$, na proporção fruto:xarope 1:2. Após a adição de 600 e 900 ppm de $\mathrm{SO}_{2}$, foram armazenados à temperatura ambiente e avaliados quanto à tendência a mudanças microbiológicas, sensoriais e físico-quimicas (Aw, pH, sólidos solúveis, acidez, açúcares redutores e não redutores, vitamina $\mathrm{C}$ e dióxido de enxofre total).

As medidas de $\mathrm{pH}$, sólidos solúveis ( $\left.{ }^{\circ} \mathrm{Brix}\right)$, acidez titulável e açúcares redutores e não redutores foram determinadas segundo os métodos do INSTITUTO ADOLFO LUTZ [8]; a umidade e o dióxido de enxofre total foram avaliados segundo os métodos da A.O.A.C. [4]; a determinação experimental (instrumental) da atividade de água das amostras foi feita em aparelho digital Aqua-Lab (da marca Decagon Devices Inc. EUA, modelo CX-2), à temperatura ambiente $28^{\circ} \pm 2^{\circ} \mathrm{C}$. O teor de vitamina $\mathrm{C}$ foi determinado pelo método espectrofotométrico citado por PEARSON [19], sendo as leituras realizadas em espectrofotômetro modelo CG UV-VIS 8000, marca Instrumentos Científicos. O produto foi avaliado microbiologicamente durante a vida-de-prateleira (mensalmente durante quatro meses) por meio das determinações microbiológicas de coliformes totais e fecais, contagem total de mesófilos, mofos e leveduras, de acordo com as recomendações do ICMSF [9].

A avaliação sensorial dos atributos de cor, sabor, aparência, textura e aceitação global dos produtos obtidos, durante a vida-de-prateleira (mensalmente durante quatro meses), foi feita através do teste de escala hedônica estruturada de 9 pontos, onde 9 representava a nota máxima "gostei muitíssimo" e 1 a nota minima "desgostei muitíssimo" [15, 16], aplicado a 30 provadores não treinados. As amostras dos produtos foram apresentadas aos provadores, à temperatura ambiente $\left(28^{\circ} \pm 2^{\circ} \mathrm{C}\right)$, servidas monadicamente em pratos plásticos codificados aleatoriamente. Os provadores posicionados em cabines individuais foram orientados a observar as caracteristicas pedidas e o preenchimento das fichas de respostas.

Os dados obtidos pelas análises físico-químicas e análise sensorial durante a vida-de-prateleira foram tratados estatisticamente através de análise de variância, teste de Tukey e teste de Ryan-Einot-Grabriel-welsch (REGWQ) pelo programa estatistico SAS (Statistical Analysis System, SAS Institute, Inc. [20]).

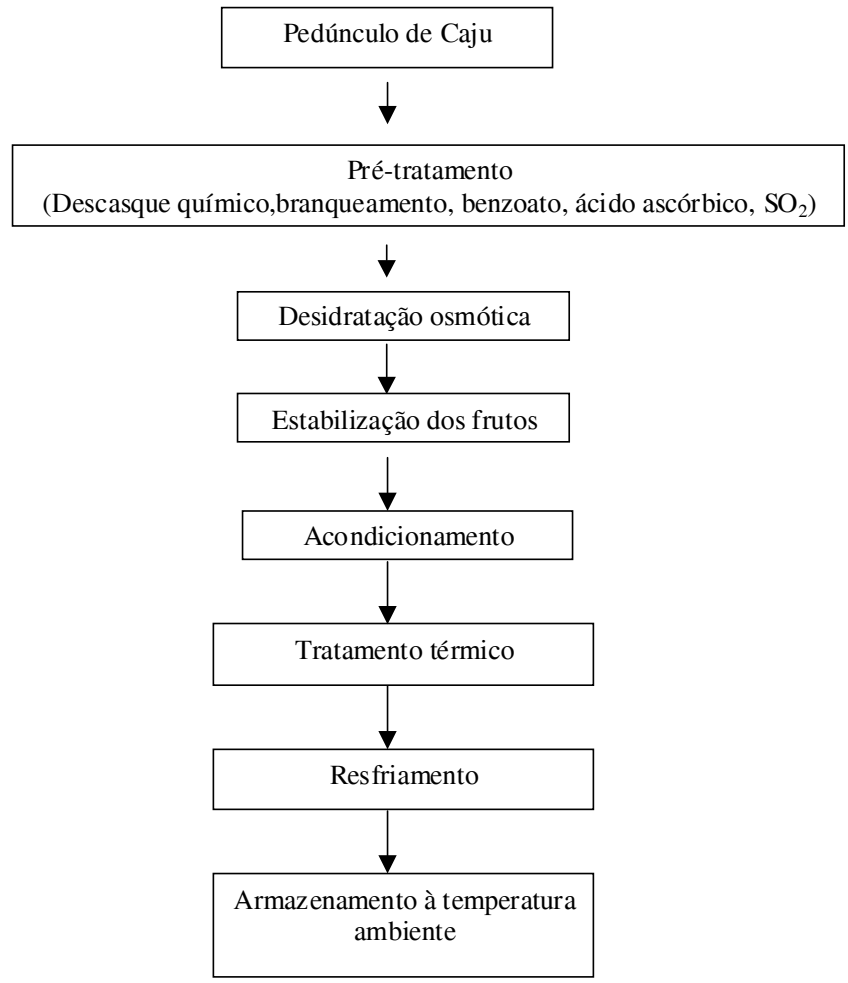

FIGURA 1. Fluxograma de conservação do pedúnculo de caju in situ por processamento mínimo/métodos combinados com emprego da tecnologia de obstáculos.

\section{3 - RESULTADOS E DISCUSSÃO}

A perda acentuada de $\mathrm{SO}_{2}$ durante a armazenagem, provavelmente por reações de degradação, mostrou a influência do tempo no processo, condições de estocagem, permeabilidade da embalagem quanto ao oxigênio e à luz, pH e conteúdo de açúcar na perda deste aditivo (Tabela 1).

TABELA 1 - Resultados das análises fĩsico-químicas da vidade-prateleira em amostras de pedúnculos de caju processados por métodos combinados durante 120 dias de armazenagem à temperatura ambiente $\left(28^{\circ} \mathrm{C} \pm 2\right)$, com teores iniciais de 600 e 900 ppm $\mathrm{SO}_{2}$.

\begin{tabular}{|c|c|c|c|c|c|c|c|c|c|c|}
\hline Tempo & Amostra & ${ }^{\circ} \mathrm{Brix}$ & PH & $\begin{array}{c}\text { Acidez } \\
\text { (\%ó́c.́ítico) }\end{array}$ & $\begin{array}{c}\text { Aç.Red. } \\
\text { (\% de glicose) }\end{array}$ & $\begin{array}{l}\text { Aç.Não Red. } \\
\text { (\%de sacarorse) }\end{array}$ & 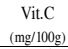 & $\begin{array}{r}\mathrm{SO}_{2} \\
(\mathrm{ppm})\end{array}$ & $\begin{array}{c}\text { Umidade } \\
(\%)\end{array}$ & Aw \\
\hline 30 & 1 & $20,0^{\mathrm{a}}$ & $3,7^{\mathrm{b}}$ & $0,35^{\mathrm{a}}$ & $17,4^{\mathrm{a}}$ & $16,9^{\mathrm{a}}$ & $49,4^{\mathrm{b}}$ & $29,0^{b}$ & $77,0^{\mathrm{a}}$ & $0,966^{6}$ \\
\hline 30 & 2 & $20,3^{\mathrm{a}}$ & $3,8^{\mathrm{a}}$ & 0, & $16,9^{\mathrm{a}}$ & $16,4^{\mathrm{b}}$ & $58,9^{\mathrm{a}}$ & $94,5^{a}$ & $76,9^{\mathrm{a}}$ & $0,963^{3}$ \\
\hline 60 & 1 & $21,7^{a}$ & $3,4^{b}$ & 0, & & $17,6^{a}$ & $26,6^{b}$ & $6,5^{b}$ & $80,9^{\mathrm{a}}$ & $0,970^{\circ}$ \\
\hline 60 & 2 & 21, & $3,5^{\mathrm{a}}$ & & 18 & $17,3^{\mathrm{a}}$ & $34,7^{a}$ & $46,8^{\mathrm{a}}$ & $78,6^{\mathrm{a}}$ & $0,968^{\mathrm{a}}$ \\
\hline 90 & 1 & 21 , & $3,4^{b}$ & 0, & 18 & $17,6^{\mathrm{a}}$ & $1,4^{a}$ & $5,6^{\mathrm{b}}$ & $77,4^{\mathrm{a}}$ & $0,975^{4}$ \\
\hline 90 & 2 & $21,2^{\mathrm{a}}$ & $3,5^{\mathrm{a}}$ & 0, & $17,1^{b}$ & $16,4^{\mathrm{a}}$ & $1,4^{a}$ & $8,7^{\mathrm{a}}$ & $77,2^{\mathrm{a}}$ & $0,9711^{\mathrm{b}}$ \\
\hline 120 & 1 & $21,6^{a}$ & $3,5^{a}$ & 0, & $17,1^{a}$ & $18,1^{a}$ & $0,1^{\mathrm{a}}$ & $1,1^{\mathrm{a}}$ & $77,1^{a}$ & $0,967^{\mathrm{a}}$ \\
\hline 120 & 2 & $21,5^{\mathrm{b}}$ & $3,5^{\mathrm{a}}$ & $0,34^{\mathrm{a}}$ & $16,6^{\mathrm{a}}$ & $18,3^{\mathrm{a}}$ & $0,4^{a}$ & $-0,8^{\mathrm{a}}$ & $77,9^{\mathrm{a}}$ & $0,966^{\natural}$ \\
\hline
\end{tabular}

- Médias dentro de uma mesma coluna com a mesma letra não diferem significativamente entre si, pelo teste de Tukey, a $\mathrm{p} \geq 0,05$. $1-600 \mathrm{ppm} \mathrm{SO}_{2}$
$2-900 \mathrm{ppm} \mathrm{SO}$

A perda da vitamina $\mathrm{C}$ durante vida-de-prateleira por 120 dias foi devida principalmente aos fenômenos difusionais e degradação quimica, que são favorecidos pela presença de luz, oxigênio, degradação dos sulfitos e condições de armazenamento. 
Os teores de umidade obtidos no transcorrer do tempo de armazenamento para o experimento estão de acordo com o valor para os alimentos processados por métodos combinados que é de 65-85\% para umidade [1].

Os resultados das análises de atividade de água para o experimento mostram que todos os valores são próximos a 0,97 , sendo, portanto, alimentos de alta umidade [22].

O aumento dos teores de açúcares redutores pode ser devido às reações de hidrólise da sacarose dentro do fruto e também em parte à adição de ácido cítrico para depressão do pH para estabilização do sistema.

Os resultados referentes à avaliação microbiológica mostraram que a contagem padrão de mesófilos em placa, mofos e leveduras e coliformes totais e fecais foram semelhantes nos dois experimentos. Estes resultados confirmam que os tipos de obstáculos usados (redução da Aw, tratamento térmico brando, redução do $\mathrm{pH}$ para 2,5, adição de ácido ascórbico, benzoato de sódio a $1000 \mathrm{ppm} \mathrm{e} \mathrm{SO}_{2}$ a 600 e $900 \mathrm{ppm}$ ) e sua intensidade foram capazes de assegurar a estabilidade microbiológica do produto durante a armazenagem à temperatura ambiente por 120 dias [14] (Tabela 2).

TABELA 2. Médias dos resultados das análises microbiológicas das amostras de pedúnculos de caju processados por métodos combinados durante 120 dias de armazenagem à temperatura ambiente $\left(28^{\circ} \mathrm{C} \pm 2\right)$, com teor inicial de 600ppm e 900ppm de $\mathrm{SO}_{2}$.

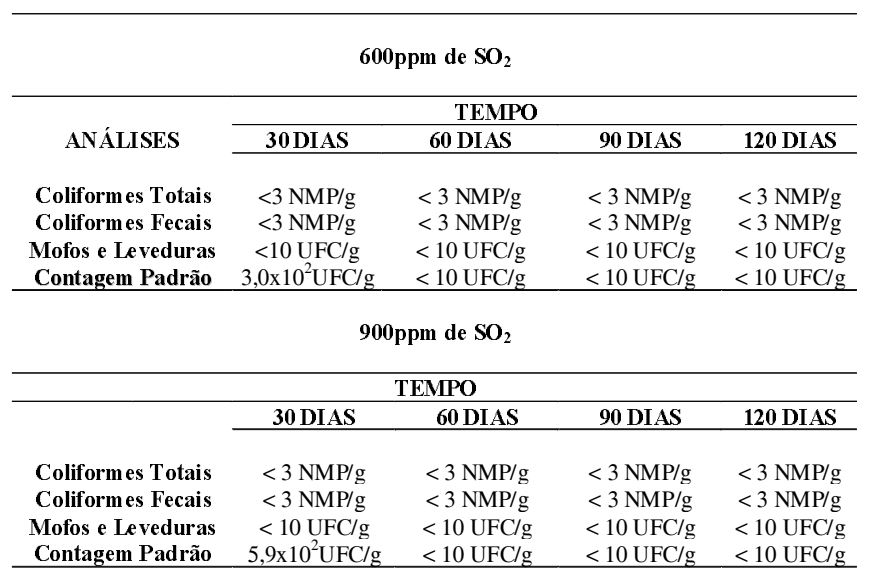

Na avaliação sensorial, o teste de aceitabilidade (escala hedônica estruturada, onde 9 é a nota máxima "gostei muitíssimo" e 1 é a nota minima "desgostei muitíssimo") apresentou os seguintes resultados:

O pedúnculo com $600 \mathrm{ppm}$ de $\mathrm{SO}_{2}$ obteve notas médias que variaram entre 7,0 (gostei moderadamente) para o atributo textura no 1 을 e 2,8 (desgostei muito) para o atributo aparência no 4ㅇ mês de armazenagem (Tabela 3), correspondendo, portanto, a um produto com boa aceitação até o segundo mês de armazenagem.

O pedúnculo com 900 ppm de $\mathrm{SO}_{2}$ corresponde a um produto com boa aceitação até o terceiro mês de armazenagem, obtendo notas médias que variaram entre 7,1 (gostei moderadamente) para o atributo sabor no 1ำ mês e 4,4 (desgostei ligeiramente) para o atributo aparência no 4ํ mês de armazenagem.

TABELA 3. Médias das notas atribuídas pelos provadores no teste de aceitabilidade (escala hedônica 1-9 pontos) dos pedúnculos de caju preservados por métodos combinados durante 120 dias de armazenagem à temperatura ambiente $\left(28^{\circ} \mathrm{C} \pm 2\right)$, com teores iniciais de 600 e $900{\mathrm{ppm} \mathrm{SO}_{2}}$

\begin{tabular}{|c|c|c|c|c|}
\hline \multicolumn{5}{|c|}{$600 \mathrm{ppm}$ de $\mathrm{SO}_{2}$} \\
\hline ATRIBUTOS & 30 DIAS & 60 DIAS & 90 DIAS & 120 DIAS \\
\hline Aparência & $6,2^{\mathrm{a}}$ & $6,1^{\mathrm{a}}$ & $4,1^{\mathrm{b}}$ & $2,8^{\mathrm{c}}$ \\
\hline Aroma & $6,6^{\mathrm{a}}$ & $6,7^{\mathrm{a}}$ & $5,1^{\mathrm{b}}$ & $5,0^{\mathrm{b}}$ \\
\hline Sabor & $6,9^{\mathrm{a}}$ & $6,8^{\mathrm{a}}$ & $6,3^{\mathrm{ab}}$ & $5,2^{\mathrm{b}}$ \\
\hline Textura & $7,0^{\mathrm{a}}$ & $6,5^{\mathrm{ab}}$ & $6,5^{\mathrm{ab}}$ & $5,7^{\mathrm{b}}$ \\
\hline Aceitacão Global & $6,9^{\mathrm{a}}$ & $6,5^{\mathrm{a}}$ & $5,9^{\mathrm{a}}$ & $4,4^{\mathrm{b}}$ \\
\hline \multicolumn{5}{|c|}{$900 \mathrm{ppm}$ de $\mathrm{SO}_{2}$} \\
\hline ATRIBUTOS & 30 DIAS & 60 DI AS & 90 DIAS & 120 DIAS \\
\hline Aparência & $6,1^{a}$ & $6,9^{\mathrm{a}}$ & $6,9^{\mathrm{a}}$ & $4,4^{\mathrm{b}}$ \\
\hline Aroma & $6,5^{\mathrm{a}}$ & $6,8^{\mathrm{a}}$ & $6,5^{\mathrm{a}}$ & $5,5^{\mathrm{b}}$ \\
\hline Sabor & $7,1^{\mathrm{a}}$ & $7,0^{\mathrm{a}}$ & $7,1^{\mathrm{a}}$ & $5,9^{\mathrm{a}}$ \\
\hline Textura & $6,5^{\mathrm{a}}$ & $6,6^{\mathrm{a}}$ & $6,9^{\mathrm{a}}$ & $6,0^{\mathrm{a}}$ \\
\hline Aceitacão Global & $6,6^{\mathrm{a}}$ & $6,7^{\mathrm{a}}$ & $7,0^{\mathrm{a}}$ & $5,2^{\mathrm{b}}$ \\
\hline
\end{tabular}

* Médias dentro de uma mesma linha com a mesma letra não são significativamente diferentes entre si, pelo teste de Tukey, a $p \geq 0,05$.

\section{4 - CONCLUSÕES}

- Os pedúnculos de caju processados por métodos combinados tratados com 900ppm de $\mathrm{SO}_{2}$ obtiveram boa aceitação sensorial, pois permaneceram por 90 dias sem escurecimento em armazenamento a temperatura ambiente. Já os pedúnculos de caju processados por métodos combinados tratados com 600ppm de $\mathrm{SO}_{2}$, somente obtiveram aceitação sensorial por 60 dias, pois após este periodo houve escurecimento dos pedúnculos prejudicando assim sua aceitação.

- Em ambos os tratamentos o processamento por métodos combinados assegurou a estabilidade microbiológica por 120 dias.

\section{5 - REFERÊNCIAS BIBLIOGRÁFICAS}

[1] ALZAMORA,S.M.; GERSCHENSON,L.N; CERRUTI,P. ; ROJAS, A.M. Shelf-stable pineapple for long-term nonrefrigereted storage. Lebensmittel- Wissenschaft und Technologie, v. 22,p. 233-236, 1989.

[2] ALZAMORA,S.M.; ARGAIZ, A., WELTI, J. Fruit preservation by combined factors. Food Res. Inter (submitted), 1992. In: AGUILERA,J.N. ; PARADA,. Cited AHI: An IberoAmerican project on intermediate moisture foods and combined methods technology. Food Res. Int. v. 25,p. 159-165, 1992.

[3] ALZAMORA, S. M.; TAPIA, M. S; ARGAIZ, A.; WELLI, J. Application of combined methods technology in minimally processed fruit. Food Res. Int., v. 22, p. 12530, 1993.

[4] A.O.A.C. Oficial methods of Analysis of the Association of Oficial Analytical Chemists. 15. ed., Arlington, p. 1158-1159, 1990.

[5] ARGAIZ,A; LOPÉZ-MALO, A.; WELTI, J. Conservación de frutas por factores combinados. I. papaya y Piña. Programa CYTED-D -V Centenário. Desarrollo de alimentos de humedad intermedia importantes para Iberoamérica. 
Subprograma Tratamiento y Conservación de Alimentos. Boletin de Divulgación de los Grupos Mexicanos, v. 4, n 9. México, 1991.

[6] BRIMELOW, C. J. B. A pragmatic approach to the development of new intermediate moisture foods. In: Properties of water in relation to quality and estability. D. Simatos \& J. L. Multon. Martinus Nijhoff, Dordrecht, The Netherlands, p. 405, 1985.

[7] FAOSTAT Database Results - FAO. Food and Agricultural Organization of the United Nations, Rome, FAO, 1998. Disponivel em WWW.URL: http;//apps 1.fao.org.

[8] INSTITUTO ADOLFO LUTZ. Normas analíticas do Instituto Adolfo Lutz. Métodos químicos e físico para análises de alimentos. 3. ed., V. 1, São Paulo, 1985. 533p.

[9] INTERNATIONAL COMISSION ON MICROBIOLOGICAL SPECIFICATION FOR FOOD. Microorganisms in foods 1. 2. ed., Toronto, 1988. 436p.

[10] JAYARAMAN,K.S. Development of intermediate moisture tropical fruits and vegetables products. Technological problems and Prospects. In: Food preservation by moisture control. Essex,UK: CC.Seow, Elsevier Applied Science, p. 175, 1988.

[11] LEISTNER, L. Hurdle technology applied to meat products of the shelf-stable product and intermediate moisture types. In: properties of water in relation to quality and stability. Simatos $\&$ J.L. MULTON, Martinus Nijhoff Publ., Dordrecht, 1985. p. 309-329.

[12] LEISTNER, L.; RODEL, W.; KRISPIEN, K. Microbiology of meat and meat products in high and intermediate moisture ranges. In: ROCKLAND, L.; ETEWART, G.F. (eds). Water activity: influences on food quality. New York: Academic Press Inc, 1981. p. 855-916.
[13] LEVI, A.; GAGEL, S.; JUVEN, B.J. Intermediate-moisture tropical fruit products for developing countries. II. Quality characteristics of papaya. J. Food Technol. v. 20,p. 163,1985 .

[14] LOPEZ-MALO, A.; PALOU, E.; WELTI, J.; CORTEZ, P. ; ARGAIZ, A. Shelf stable high moisture papaya minimally processed by combined methods. Food Research International, v. 27,p. 545-553, 1994.

[15] MONTEIRO, C.B.L. Técnicas de avaliação sensorial. 2.ed. Curitiba: UFPR/CEPPA, 1984. 101p.

[16] MORAES, M.A.C. Métodos para avaliação sensorial dos alimentos. 6.ed. Campinas, UNICAMP, 1988.

[17] OHLSSON, T. Minimal processing-preservation methods of the future: an overview. Trends in Food Science \& Technology.,v. 5, p. 341-344, 1994.

[18] PARENTE, J.I.G. et al. Diretrizes para recuperação da cajucultura no Nordeste. Fortaleza: EMBRAPA/CNPCa. p. 40, 1990 (Mimeografado).

[19] PEARSON, D. Técnicas de laboratório para el analisis de alimentos. Zaragosa: Acribia, 331 p. 1976.

[20] SAS Institute. Inc. SAS user's guide: statistics. Version 5 ed. SAS Institute, Inc., Statistical Analysis System, Cary, NC, 1985.

[21] SAJUR, S. Préconservación de duraznos por métodos combinados. MS Thesis, (Universidad Nacional de Mar del Plata, Argentina, 1985).

[22] TAPIA de DAZA, M. S.; ALZAMORA, S. M.; WELTI-CHANES, $\mathrm{J}$. Combination of preservation factors applied to minimal processing of foods. Cri. Ver. Food Sci. and Nutr., v. 36,p. 629-659, 1996. 